\title{
AUTOEFICÁCIA DOCENTE, SATISFAÇÃO E DISPOSIÇÃO PARA CONTINUAR NA DOCÊNCIA POR PROFESSORES DE EDUCAÇÃO FÍSICA
}

\author{
DR. ROBERTO TADEU IAOCHITE \\ Professor Assistente Doutor do Departamento de Educação da Universidade \\ Estadual Paulista (UNESP), Doutorado na Faculdade de Educação (UNICAMP) \\ Núcleo Estudos Avançados em Psicologia Cognitiva e Comportamental (NEAPSI/UNICAMP) \\ Núcleo de Estudos e Pesquisas em Formação Profissional no \\ Campo da Educação Física (NEPEF/UNESP) \\ (Rio Claro - São Paulo - Brasil) \\ E-mail: iaochite@rc.unesp.br \\ DR ${ }^{\mathrm{a}}$. ROBERTA GURGEL AZZI \\ Professora Assistente Doutora da Faculdade de Educação da Universidade Estadual de \\ Campinas (UNICAMP), Doutorado na Faculdade de Educação (UNICAMP) \\ Coordenadora do Núcleo Estudos Avançados em Psicologia Cognitiva e \\ Comportamental (NEAPSI/UNICAMP) \\ (Campinas - São Paulo - Brasil) \\ E-mail: betazzi@uol.com.br
}

\section{DR ${ }^{a}$. SOELY APARECIDA JORGE POLYDORO}

Professora Assistente Doutora da Faculdade de Educação da Universidade Estadual de Campinas (UNICAMP), Doutorado na Faculdade de Educação (UNICAMP)

Grupo de Pesquisa em Psicologia e Ensino Superior (PES/UNICAMP)

(Campinas - São Paulo - Brasil)

E-mail: polydoro@sigmanet.com.br

\section{DR. PEDRO JOSÉ WINTERSTEIN}

Professor Livre-Docente Aposentado da Faculdade de Educação Física da Universidade Estadual Campinas (UNICAMP), Doutorado em Ciências do Esporte (Alemanha)

(Campinas - São Paulo - Brasil)

E-mail:winterstein@fef.unicamp.br

\section{RESUMO}

Este estudo objetivou identificar e analisar a autoeficácia docente (AED) de professores de educação física e sua relação com nível de satisfação pessoal e de disposição em continuar na atividade de ensino. Participaram 220 professores de escolas públicas e privadas da região de Campinas e do Vale do Paraíba, SP. Foi utilizado uma escala de autoeficácia docente e um questionário de caracterização. A AED foi elevada, sendo que a Eficácia do manejo 
da classe mostrou-se significativamente inferior quando os professores apresentavam níveis baixos ou médios de satisfação ou de disposição. Nota-se que, apesar da tendência positiva na percepção do professor, a promoção da AED se mostra relevante, especialmente quando é discutido seu papel na prevenção ou intervenção frente à saúde do professor.

PALAVRAS-CHAVE: Autoeficácia; professor; educação física; ensino.

\section{INTRODUÇÃO}

A discussão acadêmica sobre a prática pedagógica na educação física tem recebido contribuições de diferentes referenciais teóricos no que tange à ação do professor perante aos propósitos educacionais a ela endereçados. As pesquisas sobre a ação do professor no campo da educação física têm enfocado questões relacionadas à epistemologia e especificidade do conhecimento (BETTI, 1996; BRACHT, 2003; TANI, 1996), à formação do professor (BETTI; RANGEL-BETTI, 1996; DAÓLIO, 1995; DARIDO, 2003; RANGEL-BETTI, 200I) aos conteúdos curriculares e metodologias de ensino (DARIDO, 2005; RANGEL-BETTI; GALVÃO, 2001 ; RODRIGUES; GALVÃO, 2005).

Partindo-se da premissa que a ação docente é mediada pelo pensamento do professor e suas relações com a cultura em que está inserido, portanto, por suas crenças, saberes e valores, um amplo conjunto de estudos tem sido produzido nessa direção sob as mais diversas correntes teóricas (SADALLA, I997; SADALLA; SARETTA; ESCHER, 2002; DAÓLIO, 1995).

Uma importante contribuição para essas discussões advém de estudos desenvolvidos sob a perspectiva conceitual da teoria social cognitiva (TSC), mais especificamente sob o construto da autoeficácia proposto por Albert Bandura. Dentre esses estudos, é possível citar, no cenário internacional, Woolfolk e Hoy (1990), Lin e Gorrell ( 1998), Tschannen-Moran e Woolfolk Hoy (2002), Onafowora (2005), entre outros e, no cenário nacional, aqueles realizados por Bzuneck (I 996, 2000, 2003), Pinotti (2003), Polydoro et al. (2004), Venditti Jr. (2005), laochite (2007).

A TSC desde o seu surgimento em meados da década de 1980, marcadamente pela publicação do livro Social Cognitive Theory: foundations of thought and action (BANDURA, 1986) trouxe inúmeras contribuições por meio das aplicações em diferentes áreas do conhecimento como educação, saúde, organizações, esportes.

Fundada na perspectiva da agência humana, a TSC apóia-se no princípio da reciprocidade triádica. Isso significa dizer que a visão de homem para esse referencial, é de que o indivíduo (agente) é dotado de capacidades que o possibilitam assumir o controle aos acontecimentos presentes no ambiente e não apenas reagir diante deles. Assim, o indivíduo é produtor e produto das relações que se dão entre os 
fatores determinantes presentes no ambiente (contexto), no próprio comportamento e age por meio de mecanismos de autorregulação, estabelecimento de metas e da autoeficácia.

Bandura (1993, 1997) revelou, empiricamente, que as crenças de autoeficácia afetam a escolha da ação, o esforço, a persistência e os sentimentos frente às adversidades durante a ação. Além disso, os resultados de estudos em diferentes contextos e culturas mostram que as crenças de autoeficácia são preditoras de ação subseqüente (WARE; KITSANTAS, 2007; SCHWARZER; HALLUM, 2008).

Ao investigarem o comprometimento do professor com o ensino, Ware e Kitsantas (2008) aplicaram duas escalas de autoeficácia docente e autoeficácia docente coletiva com quase 33 mil professores e diretores norteamericanos. Os resultados apontaram que ambos os construtos se mostraram relevantes e relacionados com o comprometimento e retenção dos professores com a profissão.

Num contexto cultural diferente, Schwarzer e Hallum (2008) testaram, em dois estudos experimentais, sendo um de corte longitudinal, o construto da autoeficácia docente como um possível mediador entre a ação e o estresse no trabalho. Mais de 1600 professores alemães participaram dos estudos, cujos resultados mostraram que a autoeficácia esteve relacionada como um fator de mediação entre as variáveis estudadas (entre elas, o esgotamento do professor), principalmente para professores mais jovens. Esses achados, além de reforçarem a teoria, podem revelar implicações importantes para o processo de formação continuada dos professores, sobretudo no que tange ao fortalecimento da autoeficácia e das habilidades de ensino ao longo da carreira docente.

Crenças de autoeficácia docente são entendidas como o "julgamento de suas capacidades em alcançar os resultados desejados de engajamento e aprendizagem do aluno, até mesmo entre aqueles alunos que possam ser difíceis ou desmotivados" (TSCHANNEN-MORAN; WOOLFOLK HOY, 2002, p. 783). Isso significa dizer que, nesta perspectiva, o comportamento do professor é continuamente mediado por suas convicções acerca de suas capacidades e habilidades para promover aprendizagens significativas para os alunos, ainda que em condições nem sempre favorecedoras. As consequências desse julgamento podem ser observadas direta e indiretamente no comportamento do professor e dos alunos, como por exemplo, nos níveis de esforço, persistência, interesse em ensinar, mesmo diante dos obstáculos que possam surgem no decorrer desse processo.

Considerando os desafios da prática pedagógica (carga horária excessiva, turmas numerosas, baixos salários, desrespeito, indisciplina, entre outros), é possível perguntar: o quanto os professores se julgam capazes para modificar esse contexto dado às adversidades eminentes? Qual é o nível de satisfação pessoal no exercício 
da docência? Há intenção em continuar na atividade docente? Essas e outras questões podem surgir constantemente no pensamento dos professores e o caminho escolhido para lidar com elas está diretamente relacionado às suas crenças de autoeficácia para enfrentar tais situações.

Se por um lado, as crenças favorecem ao engajamento e à motivação dos professores para vencer ou tornar as adversidades administráveis e conseguir algumas realizações, o inverso, isto é a desmotivação, o stress físico e psíquico, a exaustão, a baixa satisfação pessoal em relação ao trabalho são características que podem afetar consideravelmente as crenças de capacidades dos professores.

Com a hipótese de que um ensino eficaz não é garantia de satisfação do professor, Moe, Pazzaglia e Ronconi (2010) analisaram o papel das estratégias e interação com a práxis, o afeto positivo e autoeficácia. Com a participação de 399 professores e utilizando escalas de autoavaliação para investigar as variáveis selecionadas, encontraram que crenças de autoeficácia revelaram ter um papel mediador na relação com o afeto positivo, as estratégias de ensino e a satisfação no trabalho.

Chan et al. (2008) testaram um modelo de predição e mediação da autoeficácia docente com o comprometimento com ensino de professores asiáticos. Além da autoeficácia, experiência de ensino, percepção sobre a política organizacional da escola e diálogo reflexivo foram as variáveis investigadas. Enquanto a percepção sobre a política organizacional esteve negativamente relacionada com o compromisso dos professores, o diálogo reflexivo e a experiência de ensino estiveram relacionados positivamente. Autoeficácia docente e identificação com a escola estiveram altamente relacionadas com a mediação entre todas as variáveis do estudo.

Com o objetivo de investigar a autoeficácia docente, as relações com a satisfação pelo trabalho e a realização acadêmica dos estudantes, Caprara et al. (2006) aplicaram questionários de autorrelato para mais de 2000 professores em 75 escolas italianas de ensino médio. $\bigcirc$ desempenho dos alunos foi coletado por dois anos consecutivos. Os resultados confirmaram um modelo conceitual em que as crenças de autoeficácia docente estiveram positivamente relacionadas com a satisfação no trabalho e o desempenho dos alunos.

Na temática da satisfação do professor de educação física, estudos como os de Soriano e Winterstein (1998), Galvão (2002) e Rangel-Betti e Mizukami (1997) tem revelado que, ainda que as dificuldades não sejam poucas, nem tampouco simples essas parecem não ser suficientes para abalar a satisfação que o professor percebe com seu trabalho. Isso reforça a necessidade de investigar o que pode estar contribuindo para essa percepção.

Bandura (1997) destacou que as crenças de autoeficácia dos professores afetam tanto de modo geral quanto especíico as atividades relacionadas ao processo 
educacional. Professores que acreditam fortemente em suas capacidades se esforçam para promover nos alunos o interesse intrínseco para a aprendizagem enquanto que aqueles que apresentam insegurança na eficácia instrucional se envolvem menos com os assuntos ou atividades acadêmicas relacionadas à matéria que lecionam.

Ao apontar relações entre a autoeficácia percebida e sua relação com o comportamento de enfrentamento (ou não) pelos professores quando diante da prática pedagógica, Bandura (1997) cita a multicausalidade deste processo que segundo ele, quando os professores entram em contato com agentes estressores, aqueles com autoeficácia positiva dirigem seus esforços para resolver os problemas. Já os professores que não confiam em sua eficácia se esforçam para evitar os problemas, ao invés de se esforçarem para aliviar o distress emocional.

A relação entre as crenças de autoeficácia de professores e o contexto da educação física torna-se intrinsecamente motivador a investigação sobre a percepção desses professores sobre sua satisfação pessoal e intenção de continuidade no ensino. Uma vez que a área da educação física vem, ao longo desses últimos dez anos, passando por inúmeras transformações políticas, pedagógicas e profissionais complexas nos seus mais diversos campos de atuação. Esse cenário de mudanças pode afetar, de determinada forma, tanto o pensamento quanto a ação do professor no exercício da prática docente.

Com relação ao campo da escola, mais especificamente para o contexto das aulas de educação física, os desafios atribuídos aos professores não são poucos e nem tão pouco simples. Mudanças nos conteúdos curriculares e novas diretrizes a serem seguidas, reestruturações nos cursos de formação e capacitação profissionais, aumento nos índices de absenteísmo, sobretudo no ensino médio (GUEDES, I999; DARIDO, 2004), desmotivação por parte dos alunos para a participação nas aulas, limitado suporte do corpo administrativo da escola para a disciplina, baixo status entre os colegas de outras áreas (MARTIN; KULLINNA, 2003).

Considerando esses apontamentos, este trabalho teve como objetivos identificar e analisar a autoeficácia percebida de professores de educação física, bem como analisar sua relação com nível de satisfação pessoal e de disposição em continuar na atividade docente.

\section{MÉTODO}

\section{PARTICIPANTES}

A amostra é do tipo não-probabilística, composta por 220 professores de educação física, de ambos os sexos, matriculados em cursos de especialização em Educação Física Adaptada e Educação Física Escolar de três instituições paulistas de 
ensino superior. A faixa etária média foi de 25,4 anos. Esses professores exerciam a atividade docente em contextos de escolas públicas e privadas em diferentes municípios da região de Campinas e do Vale do Paraíba, estado de São Paulo. O convite para a participação no estudo foi feito em sala de aula durante a realização dos referidos cursos com o consentimento dos coordenadores e professores que ministravam aulas no momento da coleta de dados. Após a exposição dos objetivos do estudo, os alunos foram convidados a participar voluntariamente do estudo.

INSTRUMENTOS

Os instrumentos utilizados foram: um questionário de caracterização do participante e de sua atividade docente, composto por questões fechadas e relativas à idade, sexo, tempo de docência, tipo de escola. As variáveis "Disposição para continuar na docência" e "Satisfação pessoal com a docência" foram mensuradas por meio de uma escala, com intervalos num continuum entre I (muito baixo) a 6 pontos (muito alto), incluindo questões como "O quão satisfeito você está como docente?", "Quão disposto você está para continuar na atividade de docência". A autoefiácia docente foi avaliada pela Escala de Autoeficácia de Professores de Educação Física (POLYDORO et al., 2004). Trata-se de uma escala do tipo Lickert, com 24 itens divididos em duas dimensões representadas por duas subescalas.

A primeira é denominada de Eficácia na Intencionalidade da Ação Docente. Essa subescala possui I 4 itens (alfa de Cronbach $=0,9$ I) e representa a crença do professor na capacidade de mediar o ensino e mobilizar o estudante para a realização da s atividades. São exemplos dessa subescala: "Quanto você pode fazer para motivar os alunos que demonstram baixo interesse na atividade?", "Quanto você pode ajudar os seus alunos a darem valor à aprendizagem?", "Quanto você pode auxiliar os pais a ajudar os filhos a irem bem nas atividades escolares?". Já a segunda subescala é denominada de Eficácia no Manejo da Classe", possui 10 itens (alfa de Cronbach $=0,86$ ) e representa a crença do professor para gerenciar suas ações pedagógicas e as consequências destas para o processo de ensino. São exemplos dessa subescala: "Quanto você pode fazer para lidar com os alunos mais difíceis?", "Quão bem você pode estabelecer rotinas para manter as atividades acontecendo de maneira tranquila?". No cenário nacional, essa escala foi utilizada nos estudos de Silva, Azzi e laochite (2005), Venditti Jr (2005) e laochite (2007) e tem apresentado elevados índices de confiabilidade para a mensuração do construto em questão.

\section{PROCEDIMENTOS}

A coleta de dados ocorreu por meio de dois procedimentos de aplicação: de forma coletiva e em uma única sessão para os professores localizados em cursos de pós-graduação e de maneira individual para os professores procurados em seu 
próprio ambiente de trabalho. Após o contato inicial e explicação dos objetivos do estudo para os participantes, os instrumentos foram entregues e devolvidos após o preenchimento dos mesmos. Os diferentes procedimentos de coleta decorreram do fato de se buscar ampliar o número de professores participantes da pesquisa. Em ambos os casos, os informantes receberam uma cópia do Termo de Consentimento Livre e Esclarecido informando sobre os objetivos da pesquisa, solicitando a participação, garantindo o sigilo da identificação dos dados e a aprovação da pesquisa pelo Comitê de Ética e Pesquisa (CEP/UNITAU no 312/06). Os dados foram analisados por meio da estatística descritiva e inferencial, tendo em vista os objetivos propostos e contou com o auxílio do pacote estatístico SPSS, v. I3.0 for Windows.

\section{RESULTADOS}

A seguir são apresentados os dados referentes à percepção de autoeficácia docente dos professores de educação física. Dada a diversidade das tarefas envolvidas na ação docente, a análise das respostas foi aprofundada em termos das duas dimensões de agrupamento da autoeficácia do professor, cujos dados são mostrados na Tabela I.

Tabela I - Escores médios e desvio padrão da escala de autoeficácia de professores de educação física e das dimensões que a compõe

\begin{tabular}{lll}
\hline Dimensões & Média & Desvio Padrão \\
\hline Eficácia da intencionalidade da ação docente & 5,04 & 0,617 \\
Eficácia do manejo da classe & 4,82 & 0,680 \\
Total & 4,96 & 0,607 \\
\hline
\end{tabular}

De maneira geral, os escores demonstram uma percepção elevada de autoeficácia docente em todas as dimensões avaliadas, atingindo médias superiores em relação ao ponto médio, mesmo com a apresentação de mínimo quase na extremidade inferior da escala likert proposta no instrumento. A autoeficácia docente média observada foi de 4,96 pontos e desvio padrão de 0,60. Na dimensão Eficácia da intencionalidade da ação docente obteve-se média de 5,04 e desvio padrão de 0,61 indicando maior percepção de capacidade na mediação do processo de ensino e de mobilização do estudante para a realização da atividade. Quanto à percepção dos professores sobre sua capacidade pessoal em lidar com os múltiplos aspectos do cotidiano da aula, a dimensão Eficácia do manejo da classe, observou-se média de 4,82 e desvio padrão de 0,68 pontos.

Como pode ser visto na Tabela 2 os professores apresentaram uma tendência positiva no conjunto dos níveis muito alto, alto ou médio alto em suas respostas 
quanto à avaliação da satisfação pessoal com a docência $(87,7 \%)$ e intenção de continuidade na atividade de ensino (92,5\%). Considerando apenas os níveis alto e muito alto, os percentuais encontrados foram de $56,4 \%$ para satisfação pessoal e 66,7\% para disposição em continuar na atividade docente.

Tabela 2 - Frequência percentual dos participantes com relação aos níveis de satisfação pessoal e disposição em continuar na atividade docente

\begin{tabular}{lclll}
\hline \multirow{2}{*}{$\begin{array}{l}\text { Níveis } \\
\text { (I a 6 pontos) }\end{array}$} & Satisfação Pessoal & \multicolumn{3}{c}{ Disposição em continuar } \\
\cline { 2 - 5 } & $f$ & $\%$ & $f$ & $\%$ \\
\hline Muito alto (6) & 33 & 15,6 & 60 & 28,2 \\
Alto (5) & 86 & 40,8 & 82 & 38,5 \\
Médio alto (4) & 66 & 31,3 & 55 & 25,8 \\
Médio baixo (3) & 26 & 12,3 & 13 & 6,1 \\
Baixo (2) & - & - & 3 & 1,4 \\
Muito baixo (I) & - & - & - & - \\
Total & 211 & 100 & 213 & 100 \\
\hline
\end{tabular}

Para explorar a análise sobre a relação entre autoeficácia, satisfação e disposição, foram observadas as diferenças entre as pontuações médias obtidas na Escala conforme dois níveis de satisfação pessoal com a docência e intenção de continuidade na atividade de ensino. Considerando a pulverização das respostas nos seis níveis iniciais propostos para a avaliação e o n em cada categoria, foi realizado um agrupamento que destacasse, por um lado, as categorias que faziam referência aos níveis muito alto ou alto e por outro, as categorias relacionadas às categorias média alta, média baixa, baixo ou muito baixo. Estes resultados estão apresentados na Tabela 3.

Tabela 3 - Escores médios e desvio padrão da escala de autoeficácia de professores de educação física - geral e por dimensão - considerando as variáveis satisfação pessoal e disposição em continuar na atividade docente

\begin{tabular}{|c|c|c|c|c|c|c|}
\hline Variáveis & Dimensões & Níveis & Média & Desvio Padrão & Teste $\mathrm{t}$ & p \\
\hline \multirow{6}{*}{$\begin{array}{l}\text { Satisfação } \\
\text { pessoal }\end{array}$} & \multirow[b]{2}{*}{$\begin{array}{l}\text { Eficácia da intencionali- } \\
\text { dade da ação docente }\end{array}$} & Alto & 5,10 & 0,646 & \multirow[b]{2}{*}{0,869} & \multirow[b]{2}{*}{0,222} \\
\hline & & Médio ou Baixo & 4,99 & 0,560 & & \\
\hline & \multirow{2}{*}{$\begin{array}{l}\text { Eficácia do manejo da } \\
\text { classe }\end{array}$} & Alto & 4,90 & 0,736 & \multirow[b]{2}{*}{1,127} & \\
\hline & & Médio ou Baixo & 4,71 & 0,593 & & \\
\hline & \multirow{2}{*}{ Total } & Alto & 5,02 & 0,641 & \multirow{2}{*}{0,464} & \multirow{2}{*}{0,135} \\
\hline & & Médio ou Baixo & 4,89 & 0,554 & & \\
\hline
\end{tabular}




\begin{tabular}{|c|c|c|c|c|c|c|}
\hline Variáveis & Dimensões & Níveis & Média & Desvio Padrão & Teste $\mathrm{t}$ & p \\
\hline \multirow{6}{*}{$\begin{array}{l}\text { Disposi- } \\
\text { ção em } \\
\text { continuar }\end{array}$} & & Alto & 5,09 & 0,626 & & \\
\hline & $\begin{array}{l}\text { Eficácia da intencionali- } \\
\text { dade da ação docente }\end{array}$ & Médio ou Baixo & 4,95 & 0,593 & 0,424 & 0,130 \\
\hline & \multirow{2}{*}{$\begin{array}{c}\text { Eficácia do manejo da } \\
\text { classe }\end{array}$} & Alto & 4,90 & $0,7 \mid 8$ & \multirow{2}{*}{1,676} & \\
\hline & & Médio ou Baixo & 4,67 & 0,587 & & \\
\hline & \multirow{2}{*}{ Total } & Alto & 5,02 & 0,625 & \multirow{2}{*}{1,137} & \multirow{2}{*}{0,064} \\
\hline & & Médio ou Baixo & 4,85 & 0,557 & & \\
\hline
\end{tabular}

Nota: "Diferenças significativas $(p<0,05)$.

Como pode ser visto, os participantes apresentam uma percepção bastante positiva de sua satisfação e intenção de permanência na carreira. Observa-se que a Eficácia da intencionalidade da ação docente foi a dimensão que atingiu o maior valor, seguida pela pontuação média total. A menor média foi observada na dimensão Eficácia do manejo da classe. Os dados mostram que apesar de ainda superior ao ponto médio da escala likert, os professores indicaram dificuldades na percepção de eficácia pessoal vinculada à condição de lidar com o cotidiano da aula.

Considerando o nível de significância de $p<0,05$, apesar da média de autoeficácia ter sido sempre menor para os professores que declararam níveis de satisfação ou disposição pessoal médio ou baixo, só foram observadas diferenças estaticamente significativas entre os dois níveis quando se tratava de itens relacionados à Eficácia do manejo da classe. Isto é, entre as duas dimensões da escala, a que mais diferenciou os grupos de professores quando se considerou a satisfação pessoal e intenção de continuidade na atividade de ensino foi Eficácia do manejo da classe. A análise das pontuações obtidas pelos professores na dimensão Eficácia do manejo da classe de acordo com tais categorias, conforme a Tabela 3 sugere a associação entre baixos níveis de Eficácia da intencionalidade da ação docente, satisfação pessoal e de disposição para a atividade de ensino em educação física. Nota-se que esta dimensão obteve os menores índices de autoeficácia.

Os professores com alta satisfação pessoal com a docência mostraram-se mais auto-eficazes para lidar com o cotidiano da classe $(M=4,90)$ do que os que possuíam satisfação de níveis inferiores, médio ou baixo $(M=4,7 \mathrm{I})$. A disposição dos professores em continuar na atividade docente foi associada à maior autoeficácia para a Eficácia do manejo da classe $(M=4,90)$, em comparação com aqueles apresentavam menor disposição de persistência $(M=4,67)$. 


\section{DISCUSSÃO E CONSIDERAÇÕES FINAIS}

Os resultados indicaram níveis elevados de autoeficácia docente (4,96 numa escala de 6 pontos), fato que corrobora com outros achados na literatura em diferentes áreas do conhecimento (BZUNECK, 1996; TSCHANNEN-MORAN; WOOLFOLK HOY, 200 I ; 2002) e mais especificamente na docência em educação física (IAOCHITE, 2007; MARTIN ; KULINNA, 2003; POLYDORO et al., 2004; SILVA, AZZI, IAOCHITE, 2005; VENDITTIJR., 2005). Não se buscou nesse estudo, identificar as causas ou atribuições que justifiquem os níveis encontrados. Todavia, considerando o referencial de base, é possível argumentar em favor da relação dialógica que ao fazer julgamentos de sua capacidade, o professor estabelece com fatores presentes no ambiente e no próprio comportamento. Posto isso, é possível considerar o tempo de docência e a influência das diversas fontes de construção da autoeficácia docente - experiências diretas, persuasão, modelação e estados psicofisiológicos vividos pelos professores ao longo desse tempo.

Aprofundando essa análise por meio das dimensões do construto, estudos também revelaram uma ligeira superioridade da crença para mediar o ensino e mobilizar o estudante em relação ao manejo da classe (IAOCHITE, 2007; LOONEY, 2003; TSCHANNEN-MORAN; WOOLFOLK HOY, 2002). Relacionando esses valores com a tarefa de ensinar no contexto da educação física, gerenciar turmas numerosas, com interesses e habilidades diferentes, bem como envolver os pais nesse processo não é tarefa fácil, nem tampouco simples. Associado a isso, os obstáculos presentes no próprio ambiente/infra-estrutura da escola podem "abalar" a crença na capacidade do professor para lidar com esses aspectos.

Os níveis de satisfação com a docência e a disposição para continuar na atividade foram altos se se considerar a somatória das categorias criadas para a análise. Mais de $50 \%$ dos professores se perceberam satisfeitos com o trabalho e quase $60 \%$ deles relataram ter disposição para continuar na atividade docente. Estudos na área da educação física tem revelado resultados semelhantes (GALVÃO, 2002; RANGEL-BETTI; MIZUKAMI, 1997; SORIANO; WINTERSTEIN, 1998). A satisfação percebida pelo docente é uma das características fundamentais para o comprometimento com a tarefa de ensinar. Pela perspectiva da teoria social cognitiva, autoeficácia docente, satisfação com o trabalho e disposição em continuar ensinando podem ser vistas como determinantes recíprocas. Isso posto, justificam-se, parcialmente os resultados da relação entre as variáveis investigadas. Isto é, níveis elevados de autoeficácia, satisfação e disposição para continuar na atividade. Essa parcialidade se dá, uma vez que a autoeficácia é parte integrante de um conjunto de fatores determinantes da ação do professor. 
Olhando a relação pelo viés da saúde do professor, é evidente que não se pode afirmar que está exclusivamente relacionada à crença de autoeficácia e à satisfação pessoal. Porém, há que se considerar que essas variáveis compõem a percepção do professor na sua relação de transformação com seu ambiente, com necessidades e interesses individuais diante de uma tarefa intensamente complexa e dinâmica.

Os resultados mostraram que apesar da tendência positiva na avaliação da autoeficácia docente notaram-se, especialmente ao se observar as diferenças na percepção diante das diferentes demandas vinculadas à ação docente, como por exemplo, lidar com alunos difíceis, envolver os pais e a comunidade escolar, que ainda há possibilidade de construção de um ambiente educativo de formação inicial e continuada que seja promotor de desenvolvimento de elevadas percepções de autoeficácia para todos, professores, alunos e demais atores do processo educacional. Considerando isso, permanece importante destacar que existem outros fatores situados ao contexto profissional dos professores que potencialmente precisam ser repensados, discutidos e refletidos ainda na formação, como fatores dificultadores da prática docente.

No contexto das aulas de educação física na escola, por exemplo, estes podem ser: ter que lidar com a baixa motivação e, às vezes, desvalorização dos alunos pelo conhecimento específico da área, dificuldades relacionadas à infra-estrutura e materiais, aspectos relativos ao planejamento e administração de aulas, conteúdos e avaliação com uma carga horária semanal insuficiente, baixo status no corpo docente da instituição, além dos diferentes aspectos que interferem na vida pessoal do professor e não estão vinculados diretamente com a instituição escolar.

Moreira (1997) aponta que, em geral, as instituições de ensino consideram que o professor é naturalmente motivado e a profissão docente, por si só, proporciona o suporte à dedicação e eficiência do seu trabalho. Esta premissa precisa ser repensada, pois como foi apontado, a literatura tem trazido à luz, evidências que contradizem esta perspectiva simplista e que mostram, como disse o autor, que é difícil melhorar a qualidade do ensino sem entender quais são as expectativas, crenças, motivos e interesses que sustentam os professores na profissão docente.

Self-efficacy, satisfaction and disposition to continue in teaching practice for Physical Education Teachers

ABSTRACT: This study aimed to identify and analyze teacher self efficacy (TSE) of physical education teachers and their relationship to personal satisfaction and willingness to continue in the teaching activity. Participated 220 teachers who taught in public and private schools 
from region of Campinas and Vale do Paraíba, SP. We used a range of teaching self efficacy questionnaire and a characterization. The TSE was elevated, and the effectiveness of the management class was significantly lower when teachers had low or medium levels of satisfaction. In despite of the positive trend in the perception of teachers, promoting TSE seems to be relevant, especially when it discussed its role in prevention or intervention against the health of teachers.

KEYWORDS: Self-efficacy; teacher; physical education; teaching.

\section{La auto-eficacia, la satisfacción y disposición a seguir en la práctica docente de profesores de educación física}

RESUMEN: Este estudio tuvo como objetivo identificar y analizar la autoeficacía docente (AED) de profesores de educación física y su relación con la satisfacción personal y la voluntad de continuar en la actividad docente. 220 profesores participaron en las escuelas públicas y privadas, la región de Campinas y Vale do Paraíba, SP. Se utilizó un cuestionario de autoeficacia y outro para la caracterización. La AED fue alto, y la eficacia para la gestión de la clase fue significativamente menor cuando los profesores tenían niveles bajos o medios de satisfacción o disposición. Tenga en cuenta que, a pesar de la tendencia al alza en la percepción de los docentes, la promoción de AED parecen ser relevantes, sobre todo cuando se discute su papel en la prevención o la intervención en contra de la salud de los docentes.

PALABRAS CLAVE: Autoeficacía; docente; educación física; enseñanza.

\section{REFERÊNCIAS}

BANDURA, A. Social foundations of thought and action: a social cognitive theory. New Jersey: Prentice Hall, 1986.

Perceived self-efficacy in cognitive development and functioning. Educational Psychologist, Hillsdale, v. 28, n. 2, p. 117-148, 1993.

Self-efficacy: the exercise of control. New York: Freeman, 1997.

Social cognitive theory: an agentive perspective. Annual Review of Psychology, Palo Alto, v. 52, n. I, p. I-26, 200 I.

BETTI, I. C. R.; MIZUKAMI, M. G. N. História de vida: trajetória de uma professora de educação física. Motriz, Rio Claro, v. 3, n. 2, p. I08-I I 5, dez. 1997.

BETTI, M. Por uma teoria da prática. Motus Corporis, Rio de Janeiro, v. 3, n. 2, p. 73- I27, dez. 1996.

BRACHT, V. Educação física \& ciência: cenas de um casamento (in)feliz. ljuí: Ed. da Unijuí, 2003. 
BZUNECK, J. A. Crenças de autoeficácia de professoras do $I^{\circ}$ grau e sua relação com outras variáveis de predição e de contexto. Arquivos Brasileiros de Psicologia, Rio de Janeiro, v. 48, n. 4, p. 57-89, 1996.

. As crenças de autoeficácia do professor. In: SISTO, F.; OLIVEIRA, G. C.; FINI, L. D. Leituras de psicologia para formação de professores. Petrópolis: Vozes; Bragança Paulista: Ed. Universitária São Francisco, 2000. p. I17-134.

BZUNECK, J. A.; GUIMARÃES, S. E. R. Crenças de eficácia de professores: validação da escala de Woolfolk e Hoy. Revista Psico-USF, Bragança Paulista, v. 8, n. 2, p. I37- |43, jul./ dez. 2003.

CAPRARA, G. V. et al. Teachers' self-efficacy beliefs as determinants of job satisfaction and students' academic achievement: a study at the school level. Journal of School Psychology, New York, v. 44, n. 6, p. 473-490, dec. 2006.

CHAN, W. Y. et al. Organizational and personal predictors of teacher commitment: The mediating role of teacher efficacy and identification with school. American Educational Research Journal, Washington, v. 45, n. 3, p. 597-630, sept. 2008.

DAÓLIO, J. Da cultura do corpo. Campinas: Papirus, 1995.

DARIDO, S. C. Educação física escolar: questões e reflexões. Rio de Janeiro: GuanabaraKoogan, 2003.

A educação física na escola e o processo de formação dos não praticantes de atividade física. Revista Brasileira de Educação Física e Esporte, São Paulo, v. I8, n. I , p. 61-80, mar. 2004.

Os conteúdos da educação física na escola. In: RANGEL, I. C. A. (Coord.). Educação física na escola. Rio de Janeiro: Guanabara-Koogan, 2005. p. 64-79. GALVÃO, Z. Educação física escolar: a prática do bom professor. Revista Mackenzie de Educação Física e Esporte, São Paulo, v. I, n. I, p. 65-72, 2002.

GUEDES, D. P. Educação para a saúde mediante programas de educação física escolar. Motriz, Rio Claro, v. 5, n. I, p. 10-|4, jun. 1999.

IAOCHITE, R. T. Auto-eficácia de docentes de educação física. 2007. I 75 f. Tese (Doutorado em Educação)-Faculdade de Educação, Universidade Estadual de Campinas, Campinas, 2007.

LIN, H-L.; GORRELL, J. Pre-service teachers' efficacy beliefs in Taiwan. Journal of Research and Development in Education, Athens, v. 32, n. I, p. 17-25, 1998.

LOONEY, L. Understanding teacher's efficacy beliefs: the role of professional community. 2003. $203 \mathrm{f}$. Thesis (Doctor of Human Development)-University of Maryland, Maryland, 2003. Disponível em : <http://drum.lib.umd.edu/bitstream//903//74///dissertation.pdf>. Acesso em: 17 jan. 2007. 
MARTIN, J.; KULINNA, P. H. The development of a physical education teacher's physical activity self-efficacy instrument. Journal of Teaching Physical Education, Champaign, v. 22, n. 2, p. 2 19-232, jan. 2003.

MOE, A.; PAZZAGLIA, F; RONCONI, L. When being able is not enough: the combined value of positive affect and self-efficacy for job satisfaction in teaching. Teaching and Teacher Education, New York, v. 26, n. 5, p. I |45- I I53, jul. 2010.

MOREIRA, H. A investigação da motivação do professor: a dimensão esquecida. Educação \& Tecnologia, Curitiba, v. I, n. I, p. 74-81, jul. 1997.

ONAFOWORA, L. L. Teacher efficacy issues in the practice of novice teachers. Educational Research Quarterly, Los Angeles, v. 28, n. 4, p. 34-43, jun. 2005.

PINOTTI, G. S. A. Avaliação de práticas educativas e de auto-eficácia dos professores. 2003. 107 f. Dissertação (Mestrado em Psicologia)-Faculdade de Psicologia, Pontifícia Universidade Católica de Campinas, Campinas, 2003.

POLYDORO, S. et al. Escala de auto-eficácia docente em educação física. In: MACHADO, C. (Org.). Avaliação psicológica: formas e contextos, Braga: Psiquilíbrios, 2004. p. 330-337.

RANGEL-BETTI, I. C. Educação física e o ensino médio: analisando um processo de aprendizagem profissional. Motriz, Rio Claro, v. 7, n. I, p. 22-29, jun. 200 I.

.; BETTI, M. Novas perspectivas na formação profissional em educação física. Motriz, Rio Claro, v. 2, n. I, p. 10-15, jun. 1996.

RANGEL-BETTI, I. C.; GALVÃO, Z. Ensino reflexivo em uma experiência no ensino superior em educação física. Revista Brasileira de Ciências do Esporte, Campinas, v. 22, n. 3, p. |05- | 16, maio 200।.

RODRIGUES, L. H.; GALVÃO, Z. Novas formas de organização dos conteúdos. In: DARIDO, S. C.; RANGEL, I. C. A. (Coord.). Educação física na escola. Rio de Janeiro: GuanabaraKoogan, 2005. p. 80-102. (Série Educação Física no Ensino Superior).

SADALLA, A. M. E. A. Com a palavra, a professora: suas crenças, suas ações. 1997. I 85 f. Tese (Doutorado em Educação)-Faculdade de Educação, Universidade Estadual de Campinas, Campinas, 1997.

; SARETTA, P.; ESCHER, C. A. Análise das crenças e suas implicações para a educação. In: AZZI, R. G.; SADALLA, A. M. E. A. (Org.). Psicologia e formação docente: desafios e conversas. São Paulo: Casa do Psicólogo, 2002. p. 93-। 12.

SCHWARZER, R.; SCHMITZ, G. S. Perceived self-efficacy and teacher burnout: a longitudinal study in tem schools. Disponível em: <http://self.uws.edu.au/Conferences/2004_Schwarzer_Schmitz.pdf >. Acesso em: 7 jan. 2010. 
SCHWARZER, R.; HALLUM, S. Perceived teacher self-efficacy as a predictor of job stress and burnout: mediation analysis. Applied Psychology, East Sussex, v. 57, n. s I, p. I 52- I7I, jul. 2008. Disponível em: <http://userpage.fu-berlin.de/ health/self/se-teacher_2008.pdf>. Acesso em: 8 jan. 2010.

SILVA, A. J.; AZZI, R. G.; IAOCHITE, R. T. Auto-eficácia percebida de professores de educação física em contextos inclusivos: um estudo inicial. In: CONGRESSO INTERNACIONAL DE EDUCAÇÃO FÍSICA e MOTRICIDADE HUMANA, 4., SIMPÓSIO PAULISTA DE EDUCAÇÃO FÍSICA, I0., 2005, Rio Claro. Anais... Rio Claro: UNESP, 2005.

SORIANO, J. B.; WINTERSTEIN, P. J. Satisfação no trabalho do professor de educação física. Revista Paulista de Educação Física, São Paulo, v. 12, n. 2, p. |45-159, jul./dez. 1998.

TANI, G. Cinesiologia, educação física e esporte: ordem emanente do caos na estrutura acadêmica. Motus Corporis, Rio de Janeiro, v. 3, n. 2, p. 9-50, dez. 1996.

TSCHANNEN-MORAN, M.; WOOLFOLK HOY, A. Teacher efficacy: capturing an elusive construct. Teaching and Teacher Education, New York, v. 17, n. 7, p. 783-805, oct. 2001.

The influence of resources and support on teachers' efficacy beliefs. In: ANNUAL MEETING OF THE AMERICAN EDUCATIONAL RESEARCH ASSOCIATION, 2002, New Orleans. Proceedings... New Orleans: AERA, 2002.

VENDITTI JR., R. Análise da auto-eficácia docente de professores de educação física. 2005. 167 f. Dissertação (Mestrado em Educação Física)-Faculdade de Educação Física, Universidade Estadual de Campinas, Campinas, 2005.

WARE, H.; KITSANTAS, A. Teacher and collective efficacy beliefs as predictors of professional commitment. Journal of Educational Research, Washington, v. 100, n. 5, p. 303-310, sept./oct. 2007.

WOOLFOLK, A.E.; HOY W.K. Prospective teacher's sense of efficacy and their beliefs about control. Journal of Educational Psychology, Arlington, v. 82, n. I, p. 81-91, mars 1990.

Recebido: 01 jul. 2010 Aprovado: 20 maio 201 I

Endereço para correspondência

Roberto Tadeu laochite

Universidade Estadual Paulista, campus Rio Claro Av. 24A, 1515. Bela Vista. Rio Claro, SP. Brasil

CEP: 13.506-900. 